\title{
Forming bulges during galaxy minor mergers
}

\author{
T. J. Cox ${ }^{1}$, J. Younger ${ }^{1}$, L. Hernquist ${ }^{1}$ and P. F. Hopkins ${ }^{1}$ \\ ${ }^{1}$ Harvard-Smithsonian Center for Astrophysics, 60 Garden St., Cambridge, MA 02138, USA \\ email: tcox@cfa.harvard.edu
}

\begin{abstract}
The hierarchical formation of structure suggests that dark halos, and the galaxies they host, are shaped by their merging history. While the idea that mergers between galaxies of equal mass, i.e., major merger, produce elliptical galaxies has received considerable attention, he galaxies that result from minor merger, i.e., mergers between galaxies with a large mass ratio, is much less understood. We have performed a large number of numerical simulations of minor mergers, including cooling, star formation, and black hole growth in order to study this process in more detail. This talk will present some preliminary results of this study, and in particular, the morphology and kinematics of minor merger remnants.
\end{abstract}

Keywords. galaxies: bulges, galaxies: formation, galaxies: interaction

\section{Introduction}

The numerous talks and discussions that occurred during this five day Symposium on galactic bulges made it clear that even after several decades of observational and theoretical attention, the formation and evolution of galactic bulges is still an open question. This effort has, however, exposed a wealth of information regarding the properties of galaxy bulges which is undoubtedly linked to the processes responsible for their formation (see, e.g., Kormendy \& Kennicutt 2004).

For example, observations suggest that two classes of bulges exist. One population, termed pseudobulges, is bluer, displays rotation, has a flattened structure, and an exponential surface density. These properties have motivated an in-situ formation scenario via bars and or other galactic disk instabilities. The second population, often termed classical bulges, rotates slowly, and has a steeper $r^{1 / 4}$ surface density profile characteristic of elliptical galaxies. The resemblance between classical bulges and elliptical galaxies has motivated the notion of a common formation mechanism. The currently favored scenario is that these object are the spheroidal relics of a past major merger, i.e., a collision between galaxies that are nearly equivalent in mass.

While this merger scenario for the formation of classical bulges fits naturally into the vigorous accretion history of dark matter halos predicted by the Lambda Cold Dark Matter cosmology, there are still substantial gaps in the theoretical understanding between the dark matter merger history and the morphological properties of the galaxy at its center. In particular, the effects of mergers between galaxies with large mass ratios, so called minor mergers, are largely unknown. Further, the studies that have investigated minor mergers (see, e.g., Aguerri et al. 2001, Eliche-Moral et al. 2006) do not typically include star formation, feedback, and black hole growth. Owing to the significant role these processes are considered to play during the formation and evolution of galaxies, we have begun a large set of numerical simulations of minor mergers aimed at understanding the transformation of galaxies as a result of such events. 

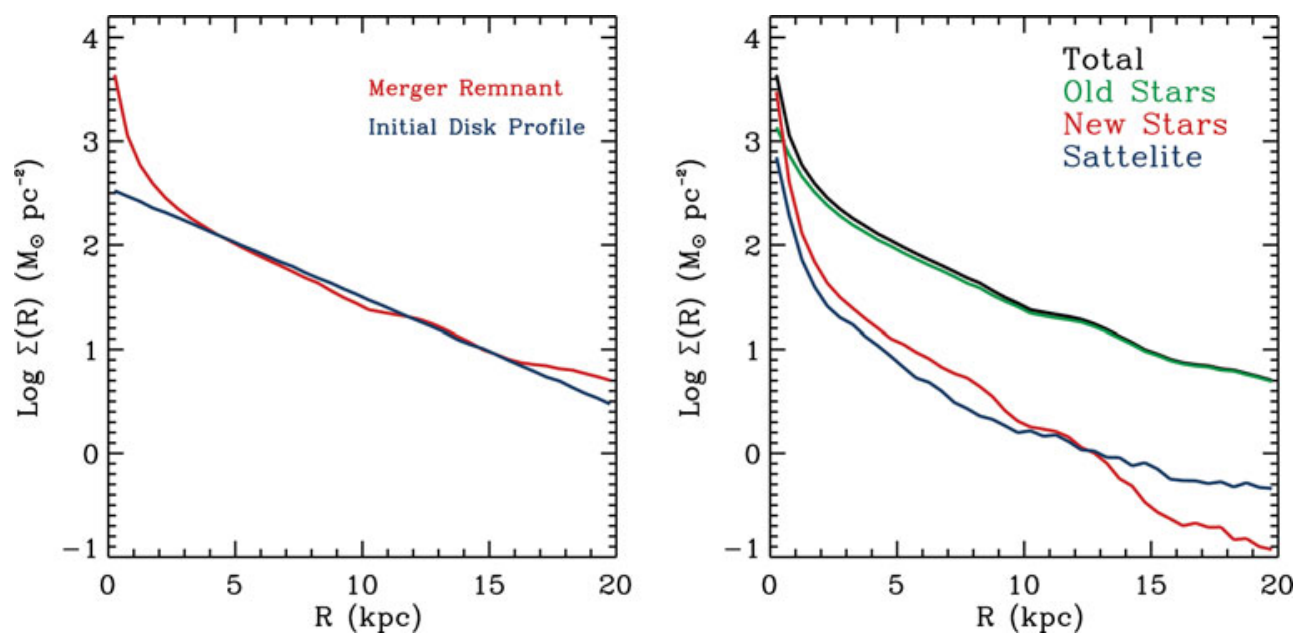

Figure 1. Surface density profile of the remnant of a 8:1 minor merger. One the left, the profile is compared to the disk of the primary, and on the right, the remnant profile is decomposed by component; old stars: those that were originally in the disk of the primary, new stars: those that were formed during the interaction, and stars that were originally part of the satellite galaxy.

\section{Methods}

To study the formation of galactic bulges during disk galaxy minor mergers we employ numerical simulations that include radiative cooling, star formation, stellar feedback, and black hole accretion. These features are implemented into a modified version of the publically available N-body/Smoothed Particle Hydrodynamics code GADGET2 (Springel 2005) that is described more completely in Springel et al. (2005b) and Cox et al. (2006).

The minor mergers we follow consist of a larger primary galaxy modeled after a Hubbletype Sb galaxy (such as the Milky Way), and a smaller satellite galaxy of one-eighth the mass modeled after a Hubble-type Im. Both galaxy models are moderately gas-rich, consisting of disk gas fractions that are 0.2 and 0.4 by mass for the Sb and Im galaxy models, respectively. The galaxy models, their structure, details of their construction, and their quiescent evolution are outlined more extensively in Younger et al. (2007).

Once the galaxy models are constructed, they are placed on a zero-energy parabolic orbit and numerically simulated until the systems merge and the remnant relaxes, which is normally $\sim 5$ Gyr. The typical evolution of such an interaction has been detailed in a number of previous studies (see, e.g., Hernquist \& Mihos 1995 and Cox et al. 2007), but generally consists of several close passages which become increasingly more radial and frequent until the final coalescence. The remnant galaxy is typically flattened and disklike, with a stellar component that is significantly thickened and dynamically hot. When gas is present, its dissipational nature dictates that it maintains a thin, cold component.

\section{Growing Bulges in Minor Mergers}

Figure 1 shows the surface density of a typical minor merger remnant. The left panel shows the remnant surface density profile in comparison to the initial surface density. The profile of the merger remnant has a disk-like structure that is similar to the primary's from $\sim 3-16 \mathrm{kpc}$, but has excessive mass at small and large radii. The excess mass at large radii is characteristic of observed anti-truncated disks and is a result of angular momentum transfer during the early stages of the interaction (see Younger et al. 2007). 

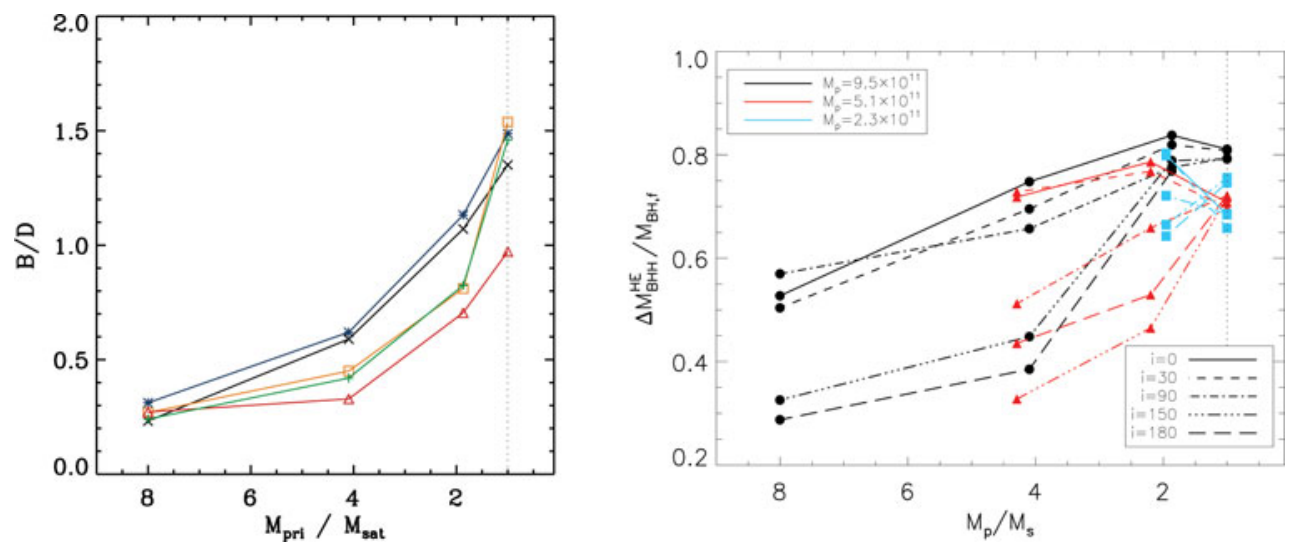

Figure 2. Properties of the minor merger remnants as a function of the primary to satellite galaxy mass ratio, $M_{\text {pri }} / M_{s a t}$. On the left, the bulge-to-disk $(B / D)$ ratio is shown, where the bulge and disk components are determined from a kinematic decomposition. On the right, the fraction of black hole growth that occurs during a high-accretion rate phase is shown. In both cases, the various lines represent runs where the satellite approaches with a number of different inclination angles with respect to the disk of the primary.

The excess surface density at small radii, however, is one of the primary characteristics of a galactic bulge, and clearly suggests that the interaction has lead to the formation of a bulge. The right panel of Figure 1 decomposes the remnant surface density profile into components as mentioned in the caption. This decomposition indicates that the central regions are composed predominantly of stars that were originally part of the primary galaxy, with an increasing fraction of stars formed during the interaction at small $(\sim 1 \mathrm{kpc})$ radii. The satellite population makes up a sub-dominant component at all radii.

We have also performed multi-component (central Sersic profile, exponential disk, and occasionally an outer exponential disk) fits to the surface density profiles of minor merger remnants, such as that shown in Figure 1, which yield estimates for the structure and mass of both the disk and bulge components.

The central concentration of new stars, which makes up a significant fraction of the bulge component, is a direct byproduct of merger-induced star formation. Numerous studies (see, e.g., Cox et al. 2007 and references therein) have shown that this radial inflow of gas and subsequent centralized star formation is the result of bar-like structures generated by the gravitational perturbation.

In addition to the bulge-disk decompositions based upon the surface density, we have also perform a kinematic analysis which delineates the bulge and disk based upon the distribution of angular momentum (disk particles have high angular momentum, bulge particle have low angular momentum). While these delineations can be tricky, this technique allows individual particles to be tagged as being part of the bulge or disk, and can therefore allow a separate characterization of each components shape and structure.

Similar to the surface density decomposition, the kinematic analysis also indicates that the merger-induced stellar population comprises a significant fraction of the galaxy bulge. In contrast, the two methods yield a different estimate of the satellite contribution to the stellar bulge. While the surface density suggests that the satellite does not contribute significantly to the bulge, the kinematics show that the stellar comonent of the satellite has very little angular momentum after it has coalesced, and therefore may form a centrally concentrated, pressure supported component of the final relaxed remnant. 
Finally, using the kinematic decomposition we can determine the growth of galaxy bulges owing to minor mergers as a function of merger mass ratio for our entire set of simulations. These results are presented in Figure 2, and clearly demonstrate that large mass ratio mergers produce systematically smaller bulges than nearly equal mass ratio mergers, a clear testimonial to the systematic elimination of rotationally supported structure owing to major galaxy mergers.

According to many theories, galaxy mergers represent the dominant mechanism to grow supermassive black holes (see, e.g., Di Matteo et al. 2005 and Hopkins et al. 2006). We have therefore also monitored the growth of a centrally located black hole during the minor mergers studied here and display the growth of the black hole during a high-accretion rate phase in the right-hand plot of Figure 2. There is a clear trend for black holes to have periods of rapid black hole growth owing to more significant merger events. Additionally, the black hole mass appears to be limited by the the feedback, consistent with feedback regulated growth and co-formation of the supermassive black hole and spheroid.

\section{Conclusions}

Using numerical simulations we have shown that the interaction and merger of unequal mass galaxies is a viable mechanism to produce galactic stellar bulges. Owing to the interaction, and specifically the merger-induced star formation, a centrally concentrated, slowly rotating stellar component is formed that is composed of both stars that are formed during the interaction, stars that were originally in the disk of the primary, and, depending upon how the bulge is quantified (surface brightness fitting or kinematics), by the accreted satellite itself.

While the results of this study support the long-standing assertion that bulges components are a natural outcome of the hierarchical formation of galaxies, further study is required to truly understand the systematic relationship between a galaxy's merger history and its final Hubble type, including the properties (shape, mean stellar age, metallicity) of its stellar bulge.

\section{Acknowledgements}

This work was supported in part by the NSF. The simulations used in this work were performed at the Center for Parallel Astrophysical Computing at Harvard-Smithsonian Center for Astrophysics.

\section{References}

Aguerri J. A. L., Balcells M., \& Peletier R. F., 2001, A\&A, 367, 428

Cox T. J., Dutta S., Di Matteo T., Hernquist L., Hopkins P. F., Robertson B., \& Springel V., 2006, ApJ, 650, 791

Cox T. J., Jonsson P., Primack J. R., Somerville R. S., \& Dekel A., 2007, submitted to MNRAS

Di Matteo T., Springel V., \& Hernquist L., 2005, Nature, 433, 604

Eliche-Moral M. C., Balcells M., Aguerri J. A. L., \& González-García A. C., 2006, A\&A, 457, 91

Hernquist, L., \& Mihos, J. C., 1995, ApJ, 448, 41

Hopkins P. F., Hernquist L., Cox T. J., Di Matteo T., Robertson B., \& Springel V., 2006, ApJS, 163,1

Kormendy, J. \& Kennicutt, Jr., R. C., 2004, ARA\&A, 42, 603

Springel, V. 2005, MNRAS, 364, 1105

Springel V., Di Matteo T., \& Hernquist L., 2005b, MNRAS, 361, 776

Younger J. D., Cox T. J., Seth A. C., \& Hernquist L., 2007, ApJ accepted (astro-ph/0707.4481) 